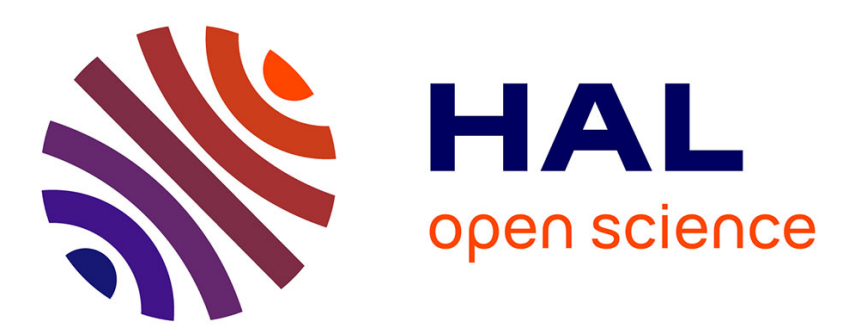

\title{
Acoustic impedance of micro perforated membranes: Velocity continuity condition at the perforation boundary
}

\author{
Chenxi Li, Ben Cazzolato, Anthony Zander
}

\section{- To cite this version:}

Chenxi Li, Ben Cazzolato, Anthony Zander. Acoustic impedance of micro perforated membranes: Velocity continuity condition at the perforation boundary. Journal of the Acoustical Society of America, 2016, 139 (1), pp.93-103. 10.1121/1.4939489 . hal-02345729

\author{
HAL Id: hal-02345729 \\ https://hal.science/hal-02345729
}

Submitted on 4 Nov 2019

HAL is a multi-disciplinary open access archive for the deposit and dissemination of scientific research documents, whether they are published or not. The documents may come from teaching and research institutions in France or abroad, or from public or private research centers.
L'archive ouverte pluridisciplinaire HAL, est destinée au dépôt et à la diffusion de documents scientifiques de niveau recherche, publiés ou non, émanant des établissements d'enseignement et de recherche français ou étrangers, des laboratoires publics ou privés. 


\title{
Acoustic impedance of micro perforated membranes: Velocity continuity condition at the perforation boundary
}

\author{
Chenxi Li, ${ }^{a)}$ Ben Cazzolato, and Anthony Zander \\ School of Mechanical Engineering, The University of Adelaide, Adelaide, South Australia 5005, Australia
}

\begin{abstract}
The classic analytical model for the sound absorption of micro perforated materials is well developed and is based on a boundary condition where the velocity of the material is assumed to be zero, which is accurate when the material vibration is negligible. This paper develops an analytical model for finite-sized circular micro perforated membranes (MPMs) by applying a boundary condition such that the velocity of air particles on the hole wall boundary is equal to the membrane vibration velocity (a zero-slip condition). The acoustic impedance of the perforation, which varies with its position, is investigated. A prediction method for the overall impedance of the holes and the combined impedance of the MPM is also provided. The experimental results for four different MPM configurations are used to validate the model and good agreement between the experimental and predicted results is achieved.
\end{abstract}

\section{INTRODUCTION}

Micro perforated panels (MPPs) are thin panels perforated with sub-millimetre sized holes, and have been used in noise control for decades as an alternative to conventional porous materials. When backed with an air cavity and a rigid wall, the MPP shows effective sound absorption, and this combined structure is called a micro perforated absorber (MPA). Although its sound absorbing bandwidth is relatively narrow compared to a porous material of similar thickness, the MPA is favoured for its unique properties. Unlike porous materials, MPPs are used in hospitals and electronic industries where particles must be avoided (Pfretzschner et al., 2006). Metal MPPs can be used in harsh conditions instead of porous materials. For example, they are used inside the engines of cars and aircraft due to their resistance to high temperature. In addition, the analytical model for the prediction of the sound absorption of MPPs is well developed, which offers the opportunity to design MPPs to control specific sources of noise (Maa, 1998).

The classical analytical model for the prediction of the sound absorption and acoustic impedance of MPPs was developed by Maa (1975) and has been widely used since that time. In recent years, Wang et al. (2010) investigated the sound absorption of an MPP backed by an irregularshaped cavity based on Maa's theory. Using the classical theory, Liu and Herrin (2010) investigated partitioning the backing cavity of the MPA to enhance the absorption of normally incident plane waves. When Ruiz et al. (2011) investigated the sound absorption of multiple-layer MPPs, the acoustic impedance of each layer was obtained using the classical theory. Based on Maa's model, Park (2013) also analysed the combination of a MPP and a Helmholtz resonator. Herdtle et al. (2013) extended Maa's theory for micro

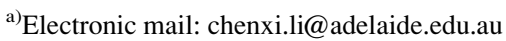

perforated materials with tapered holes. However, these studies are all based on Maa's classical model and neglect the effect of the panel vibration.

Maa's classical model assumes the panel to be rigid and as a consequence the effect of the panel vibration is neglected. However, additional sound absorbing peaks, which are not observed in Maa's model, are observed in the low frequency range of experimental results (Toyoda et al., 2010). These unexpected peaks are evidence of the effect of panel vibration on the acoustic impedance of the MPA. This effect could be very significant when the panel is very light and thin, or if membranes are used in the form of a microperforated membrane (MPM).

To investigate this effect of the panel/membrane vibration on the acoustic impedance of an MPP/MPM, Kang and Fuchs (1999) coupled the acoustic impedance of a limp membrane with the acoustic impedance due to the perforations and derived an expression for the total acoustic impedance of an infinite MPM. They achieved this using the electric-acoustic analogy to combine the acoustic impedance due to the structural vibration with the acoustic impedance of the perforations as predicted by Maa's model. Thus, the effect of the size of the membrane was neglected in their model. Similarly, Lee et al. (2005) investigated the acoustic impedance of a flexible rectangular MPP, where the finite size of the panel was considered based on a modal approach. Bravo et al. (2012) extended the method of Lee et al. (2005) to a circular MPP. Takahashi and Tanaka (2002) coupled the acoustic impedances due to the MPP vibration and the perforations by spatially averaging the flow velocity through the perforations.

Note that all these methods used Maa's model to calculate the acoustic impedance due to the perforations, assuming that the vibration of the panel/membrane has no effect on the acoustic impedance of the perforations. In Maa's (1975) classical model, the particle velocity at the hole wall 
boundary is assumed to be zero. Although Takahashi and Tanaka (2002) investigated the relative velocity at the airsolid interface in the perforation, their alternative method to couple the acoustic impedances due to the perforations and the plate vibration still calculated the acoustic impedance due to the perforation using Maa's theory based on the rigid wall assumption. However, if the panel/membrane vibration is significant, the particles at the hole wall boundary adhere to the hole wall due to the no-slip boundary condition and their velocity can be assumed to be equal to the panel/membrane vibration velocity. Hence, the acoustic impedance due to the perforation is not constant as in Maa's model but varies depending on the position of the hole on the panel/ membrane surface. The spatially varying acoustic impedance implies that for thin membranes for which vibration is not negligible, the membrane vibration could have a significant effect on the acoustic impedance of the perforation, which has been neglected in previous research. This no-slip boundary condition which assumes the particle velocity at the perforation wall boundary is equal to the vibration velocity of the material, and the spatially varying acoustic impedance of MPMs is previously unexplored.

This paper aims to investigate the acoustic impedance and sound absorption of a finite-sized circular MPM under tension using a new boundary condition which assumes that the velocity of the air particles at the hole wall boundary are equal to the vibration velocity of the membrane surrounding the hole. The new no-slip boundary condition is introduced in Sec. II A. Based on this new boundary condition, an expression for the variable acoustic impedance of the perforation is obtained. In this expression, the vibration velocity of the membrane remains unknown. The vibration velocity and the acoustic impedance of the circular membrane are investigated in Sec. II B. In the developed model, the hole diameter and the perforation ratio are assumed to be sufficiently small that the effect of the holes on the motion of the membrane can be neglected. The acoustic impedance of holes located in different positions is compared in Sec. II C. The overall impedance of the holes is derived and compared with that of Maa's classic model in Sec. II D. To validate the developed model, sound absorption experiments were carried out on four different MPMs and the experimental results are compared with the model predictions in Sec. III. Good agreement is obtained between the experimental and the predicted results for three of the MPM samples, and demonstrates the accuracy of this model. To further investigate the proposed model, four additional MPMs were manufactured and the effect of the hole position on the sound absorption of MPMs was studied in Sec. IIIC. The good agreement achieved for the four additional MPMs also validates the proposed model.

\section{ANALYTICAL MODELING}

In Sec. II A, an analytical model using the proposed boundary condition is derived. The derivation starts with the motion equation of air particles in a small hole. The solutions of this equation using the conventional and the proposed boundary condition are compared theoretically. The expressions for the acoustic impedance due to each of the perforations and for the combined MPM are also presented.

\section{A. Acoustic impedance and boundary condition of flexible MPMs}

\section{Motion equation of air particles in a small hole}

When a sound wave is traveling through the small hole of a MPM or panel with a hole radius of $r_{0}$, the particle velocity $v$ is a function of the distance, $r$, from the centre of the hole to the position of the specific air particle, as shown in Fig. 1. This relationship between the particle velocity in the hole and the sound pressure applied on the membrane or panel surface is governed by the motion equation of the air particle (Maa, 1975),

$$
\left(\frac{\partial^{2}}{\partial r^{2}}+\frac{1}{r} \frac{\partial}{\partial r}+K_{\text {air }}^{2}\right) v(r)=-\frac{\Delta p}{\mu h},
$$

where $K_{\text {air }}^{2}=-j\left(\rho_{0} \omega / \mu\right), \omega$ denotes the angular frequency and is equal to $2 \pi f$, where $f$ is the frequency, $\mu$ denotes the dynamic viscosity of air, $\rho_{0}$ denotes the density of air, $\Delta p$ denotes the pressure difference applied between the front and back surfaces of the membrane/panel, $r$ denotes the radial coordinate relative to the local coordinates of each perforation, and $h$ denotes the thickness of the membrane/ panel, which is also the length of the hole. Equation (1) is an inhomogeneous differential equation and its general solution is

$$
v(r)=A J_{0}\left(K_{\mathrm{air}} r\right)-\frac{\Delta p}{\mu h K_{\mathrm{air}}^{2}},
$$

where $J_{0}$ is the Bessel function of the first kind and zero order and the constant $A$ can be obtained by applying the appropriate boundary condition.

\section{The conventional rigid wall boundary condition}

To solve Eq. (2), it is necessary to determine the boundary condition. Due to the effect of viscosity, the air particles at the hole wall boundary adhere to the hole wall and their velocities are equal to the vibration velocity of the material.

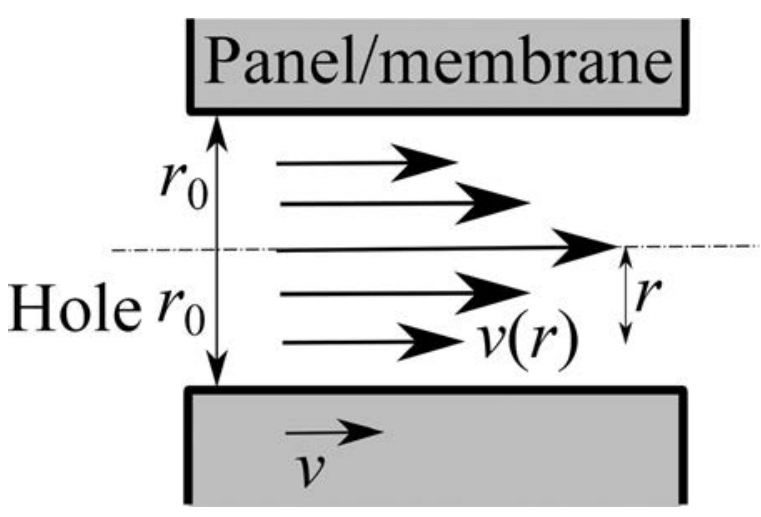

FIG. 1. Particle velocity $v(r)$ in a hole of radius $r_{0}$ of the MPM or panel as a function of the distance, $r$, from the perforation axis in the local coordinate of each perforation. 
Maa $(1975,1997)$ assumed that the panel vibration due to the incident sound pressure is negligible and the panel can therefore be considered to be rigid, i.e.,

$$
v\left(r=r_{0}\right)=0 \text {. }
$$

Based on Maa's assumption, the particle velocity as a function of radius is obtained as

$$
v(r)=\frac{-\Delta p}{h \mu K_{\mathrm{air}}^{2}}\left[1-\frac{J_{0}\left(K_{\mathrm{air}} r\right)}{J_{0}\left(K_{\mathrm{air}} r_{0}\right)}\right],
$$

and the average velocity over the hole area is expressed as

$$
\bar{v}=\frac{\int_{0}^{r_{0}} v(r) 2 \pi r d r}{\pi r_{0}^{2}}=\frac{\Delta p}{j \omega \rho_{0} h}\left[1-\frac{2}{K_{\mathrm{air}} r_{0}} \frac{J_{1}\left(K_{\mathrm{air}} r_{0}\right)}{J_{0}\left(K_{\mathrm{air}} r_{0}\right)}\right],
$$

where $J_{1}$ is the Bessel function of the first kind and first order. The normalized acoustic impedance is therefore given by

$$
z=\frac{\Delta p}{\bar{v} \rho_{0} c_{0} \delta}=\frac{j \omega \rho_{0} h}{\rho_{0} c_{0} \delta}\left[1-\frac{2}{K_{\mathrm{air}} r_{0}} \frac{J_{1}\left(K_{\mathrm{air}} r_{0}\right)}{J_{0}\left(K_{\mathrm{air}} r_{0}\right)}\right]^{-1},
$$

where $c_{0}$ is the sound speed in air and $\delta$ is the perforation ratio of the panel. When an end correction for the hole is considered, Eq. (6) is rewritten as (Maa, 1997)

$$
\begin{aligned}
z_{\text {Maa }}= & \frac{0.147}{d^{2}} \frac{h}{\delta}\left(\sqrt{1+\frac{x^{2}}{32}}+\frac{\sqrt{2}}{8} \frac{x d}{h}\right) \\
& +0.294 \times 10^{-3} \frac{j \omega h}{\delta}\left(1+\frac{1}{\sqrt{9+\frac{x^{2}}{2}}}+0.85 \frac{d}{h}\right),
\end{aligned}
$$

where $d$ is the diameter of the holes and equal to $2 r_{0}$, and $x$ is called the perforation constant and is expressed as $d \sqrt{f / 10}$.

Equation (7) is widely used to calculate the acoustic impedance of MPPs and is reported to show accurate agreement with experimental results. It should be noted that Eq. (7) is based on the assumption that the panel vibration velocity is equal to zero, and is valid only when the panel vibration is negligible and the panel can be assumed rigid.

\section{The proposed boundary condition}

For lightweight MPMs, to be considered henceforth, the vibration of the membrane is significant and needs to be considered. Therefore, the proposed boundary condition between the membrane motion and the air in the hole can be expressed as

$$
v\left(r=r_{0}\right)=v_{\text {membrane }},
$$

where $v_{\text {membrane }}$ denotes the vibration velocity of the membrane. Substituting Eq. (8) into Eq. (2) gives

$$
A=\frac{v_{\text {membrane }}+\frac{\Delta p}{\mu h K_{\text {air }}^{2}}}{J_{0}\left(K_{\text {air }} r_{0}\right)} .
$$

Substituting Eq. (9) into Eq. (2), the particle velocity is obtained as

$$
v(r)=v_{\text {membrane }} \frac{J_{0}\left(K_{\mathrm{air}} r\right)}{J_{0}\left(K_{\mathrm{air}} r_{0}\right)}-\frac{\Delta p}{h \mu K_{\mathrm{air}}^{2}}\left[1-\frac{J_{0}\left(K_{\mathrm{air}} r\right)}{J_{0}\left(K_{\mathrm{air}} r_{0}\right)}\right] .
$$

Integrating over the area of the hole yields the average particle velocity

$$
\begin{aligned}
\bar{v}= & \frac{\int_{0}^{r_{0}} v(r) 2 \pi r d r}{\pi r_{0}^{2}} \\
= & v_{\text {membrane }} \frac{2}{K_{\mathrm{air}} r_{0}} \frac{J_{1}\left(K_{\mathrm{air}} r_{0}\right)}{J_{0}\left(K_{\mathrm{air}} r_{0}\right)} \\
& +\frac{\Delta p}{j \omega \rho_{0} h}\left[1-\frac{2}{K_{\mathrm{air}} r_{0}} \frac{J_{1}\left(K_{\mathrm{air}} r_{0}\right)}{J_{0}\left(K_{\mathrm{air}} r_{0}\right)}\right] .
\end{aligned}
$$

Therefore, the normalized acoustic impedance of a single hole is expressed as

$$
z_{\text {hole }}=\frac{\Delta p}{\bar{v} \rho_{0} c_{0}}=\frac{1}{\rho_{0} c_{0} \frac{v_{\text {membrane }}}{\Delta p} \frac{2}{K_{\text {air }} r_{0}} \frac{J_{1}\left(K_{\text {air }} r_{0}\right)}{J_{0}\left(K_{\text {air }} r_{0}\right)}+\frac{\rho_{0} c_{0}}{j \omega \rho_{0} h}\left[1-\frac{2}{K_{\text {air }} r_{0}} \frac{J_{1}\left(K_{\text {air }} r_{0}\right)}{J_{0}\left(K_{\text {air }} r_{0}\right)}\right]} .
$$

Comparing Eq. (12) with Eq. (6), it could be observed that the factor $\left(1 / j \omega \rho_{0} h\right)\left[1-\left(2 / K_{\text {air }} r_{0}\right)\left[J_{1}\left(K_{\text {air }} r_{0}\right) / J_{0}\left(K_{\text {air }} r_{0}\right)\right]\right]$ in Eq. (12) is similar to Eq. (5). This similarity implies that this factor represents the average particle velocity of a hole under Maa's rigid wall assumption. Therefore, Eq. (12) can be rewritten as

$$
z_{\text {hole }}=\frac{1}{\rho_{0} c_{0} \frac{v_{\text {membrane }}}{\Delta p} \frac{2}{K_{\text {air }} r_{0}} \frac{J_{1}\left(K_{\text {air }} r_{0}\right)}{J_{0}\left(K_{\text {air }} r_{0}\right)}+\frac{1}{z_{\text {Maa }} \delta}}=\frac{1}{\frac{2}{K_{\text {air }} r_{0}} \frac{J_{1}\left(K_{\text {air }} r_{0}\right)}{J_{0}\left(K_{\text {air }} r_{0}\right)} \frac{1}{z_{\text {membrane }}}+\frac{1}{z_{\text {Maa }} \delta}},
$$


where $z_{\text {membrane }}$ denotes the normalized acoustic impedance of the membrane, which can be obtained from the motion equation of membrane vibration, and $z_{\text {Maa }} \delta$ denotes the acoustic impedance of a single hole under Maa's rigid wall assumption. If Eq. (7) is used to calculate this impedance, the end correction for the hole is included.

Equation (13) implies that the acoustic impedance of an MPM hole is a function of the acoustic impedance of the hole under the rigid wall assumption and the acoustic impedance of the membrane vibration in the vicinity of the hole. If it is a limp membrane, the membrane vibration velocity is a constant over the membrane surface when excited by a plane wave and the overall normal acoustic impedance of the MPM is obtained by combining the constant impedance due to the membrane vibration and the impedance due to the perforations. However, in acoustic engineering projects, membrane materials are commonly fixed on a rigid frame. Hence, the finite boundary condition of the fixed edge and the tension due to the stretching of the membrane affect the acoustic impedance of the membrane. This acoustic impedance of the finite sized membrane under tension will be a function of the position, as shown in Fig. 2. Therefore, the acoustic impedance obtained from Eq. (13) also varies depending on the position coordinates.

\section{B. Motion equation and impedance of membranes considering the viscosity effects on the hole walls}

In Eq. (13), the vibration velocity of the membrane $v_{\text {membrane }}$ is unknown. It is assumed in this paper that the hole diameter and the perforation ratio are sufficiently small such that the mechanical properties of the membrane (that is, the effective surface density and stiffness) are unaffected by the presence of the perforations (Burgemeister and Hansen, 1996). When a circular membrane is fixed on a circular rim with a radius of $R_{0}$ and driven by a sound pressure $\Delta p$, its motion equation in a polar coordinate system is given by (Kinsler et al., 1999)

$$
T \nabla^{2} \xi(R)+\omega^{2} \rho_{\mathrm{p}} \xi(R)=-\Delta p,
$$

where $T$ is the tension per unit length applied on the membrane surface, $\xi(R)$ is the membrane displacement, $R$

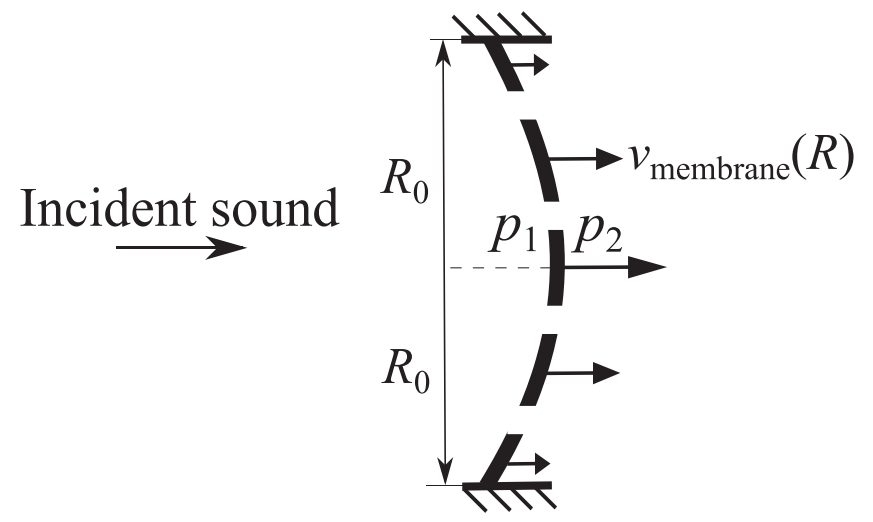

FIG. 2. Distribution of the membrane vibration velocity (vibrating in the fundamental mode). denotes the radial position coordinate on the membrane surface, which has a maximum value at the radius of the membrane $R_{0}, \rho_{\mathrm{p}}$ is the surface density of the membrane, and $\Delta p=p_{1}-p_{2}$.

As in the case of a string (Walstijn, 2009), the internal damping plays an important part in the vibration of a membrane. Extending the expression of the internal damping of a string in the work of Walstijn (2009) to that of a membrane, Eq. (14) may be rewritten as

$$
T \nabla^{2} \xi(R)+2 j \omega \eta \nabla^{2} \xi(R)+\omega^{2} \rho_{\mathrm{p}} \xi(R)=-\Delta p,
$$

where $\eta$ is the internal damping ratio of the membrane. Note that the damping is related to the curvature of the tensioned, circular membrane in this work, which differs from the conventional expression of complex tension $T \times(1+j \eta)$ as seen in the work of Song and Bolton (2003) and the book of Kinsler et al. (1999).

The general solution of Eq. (15) is given by

$$
\xi(R)=B J_{0}\left(K_{\mathrm{mem}} R\right)-\frac{\Delta p}{\omega^{2} \rho_{\mathrm{p}}},
$$

where $K_{\mathrm{mem}}^{2}=\omega^{2} \rho_{\mathrm{p}} /(T+2 j \omega \eta)$ and the constant $B$ depends on the boundary condition of the membrane vibration $\xi\left(R=R_{0}\right)=0$. Applying this boundary condition yields

$$
B=\frac{\Delta p}{\omega^{2} \rho_{\mathrm{p}}} \frac{1}{J_{0}\left(K_{\mathrm{mem}} R_{0}\right)}
$$

and

$$
\xi(R)=\frac{\Delta p}{\omega^{2} \rho_{\mathrm{p}}}\left[\frac{J_{0}\left(K_{\mathrm{mem}} R\right)}{J_{0}\left(K_{\mathrm{mem}} R_{0}\right)}-1\right] .
$$

Hence, the velocity varying with the radial coordinate is given by

$$
v_{\text {membrane }}(R)=j \omega \xi(R)=j \omega \frac{\Delta p}{\omega^{2} \rho_{\mathrm{p}}}\left[\frac{J_{0}\left(K_{\mathrm{mem}} R\right)}{J_{0}\left(K_{\mathrm{mem}} R_{0}\right)}-1\right],
$$

and the corresponding normalized acoustic impedance is expressed as

$$
\begin{aligned}
z_{\text {membrane }}(R) & =\frac{\Delta p}{\rho_{0} c_{0} v_{\text {membrane }}(R)} \\
& =\frac{\omega^{2} \rho_{\mathrm{p}}}{\rho_{0} c_{0} j \omega}\left[\frac{J_{0}\left(K_{\mathrm{mem}} R\right)}{J_{0}\left(K_{\mathrm{mem}} R_{0}\right)}-1\right]^{-1} .
\end{aligned}
$$

Integrating over the surface of the membrane and dividing by the membrane area, $\pi R_{0}^{2}$, yields the space average vibration velocity and the space average normalized acoustic impedance

$$
\bar{v}_{\text {membrane }}=j \omega \frac{\Delta p}{\omega^{2} \rho_{\mathrm{p}}}\left[\frac{2}{K_{\mathrm{mem}} R_{0}} \frac{J_{1}\left(K_{\mathrm{mem}} R_{0}\right)}{J_{0}\left(K_{\mathrm{mem}} R_{0}\right)}-1\right]
$$

and 


$$
\bar{z}_{\text {membrane }}=\frac{\omega \rho_{\mathrm{p}}}{j \rho_{0} c_{0}}\left[\frac{2}{K_{\mathrm{mem}} R_{0}} \frac{J_{1}\left(K_{\mathrm{mem}} R_{0}\right)}{J_{0}\left(K_{\mathrm{mem}} R_{0}\right)}-1\right]^{-1} .
$$

\section{Acoustic impedance of each perforation of a circular MPM}

The acoustic impedance of a hole in a circular MPM as a function of its radius is expressed by Eq. (13). Substituting Eq. (20) into Eq. (13) gives the acoustic impedance of a hole as

$$
z_{\text {hole }}(R)=\frac{1}{\frac{2 J_{1}\left(K_{\text {air }} r_{0}\right) \rho_{0} c_{0} j}{K_{\text {air }} r_{0} J_{0}\left(K_{\text {air }} r_{0}\right)} \frac{J_{0}\left(K_{\mathrm{mem}} R\right)}{\omega \rho_{\mathrm{p}}}\left[\frac{J_{0}\left(K_{\mathrm{mem}} R_{0}\right)}{J_{\text {maa }}}-1\right]+\frac{1}{z_{\mathrm{Ma}} \delta}},
$$

where Eq. (23) is a function of the radial coordinate $R$, which is related to the membrane geometry (not the radial coordinate $r$ of the air particle in the hole). The effect of the hole position on the hole impedance can be predicted using Eq. (23), although it is non-linear and is therefore difficult to investigate analytically. Therefore, an example is utilised here, where a circular MPM with surface density $\rho_{\mathrm{p}}=0.25 \mathrm{~kg} / \mathrm{m}^{2}$, is stretched under a tension $T=125 \mathrm{~N} / \mathrm{m}$ and its internal damping ratio is $\eta=0.02$. It is fixed on a rigid ring with a radius of $R_{0}=50 \mathrm{~mm}$. The perforation parameters are: hole radius $r_{0}=0.0292 \mathrm{~mm}$; membrane thickness $h=0.17 \mathrm{~mm}$; backing cavity depth $D=25 \mathrm{~mm}$; perforation ratio $\delta=0.15 \%$. The resistances and reactances of five holes at different radii calculated using Eq. (23), normalized by $\rho_{0} c_{0}$, are shown in Figs. 3(a) and 3(b). The radial coordinate $R$ of these holes varies from $R=10$ to $R=50 \mathrm{~mm}$ and has been chosen to show the variability of the perforation impedance. The normalized resistances and reactances of a hole calculated by Maa's classic model [Eq. (7)] are also shown in these figures. Note that when $R=50 \mathrm{~mm}, J_{0}\left(K_{\mathrm{mem}} R_{0}\right) / J_{0}\left(K_{\mathrm{mem}} R_{0}\right)=1$ and the prediction of Eq. (23) is consistent with that of Eq. (7) and thus Maa's theory.

It can be concluded from Figs. 3(a) and 3(b) that the acoustic impedance of a hole near the centre of the membrane is more significantly affected by the membrane motion than that of a hole near the edge of the membrane. This is because the membrane elements near the centre vibrate more significantly than those near the membrane edge.

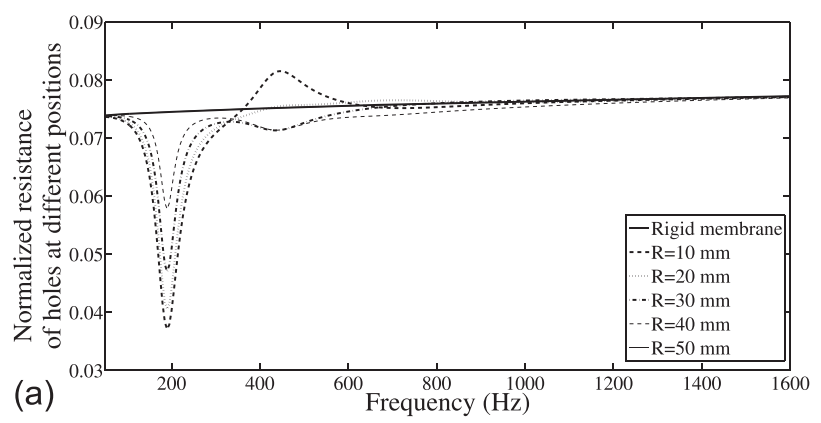

In addition, the effects of the membrane vibration on the perforation impedance occur mainly in the low frequency range where the tension and the internal damping affect the membrane impedance significantly. In contrast, the surface density governs the membrane impedance in the high frequency range (mass controlled) and no significant effect of the membrane vibration on the perforation impedance is observed for a constant surface density.

\section{Combined acoustic impedance of the MPM system}

In Sec. II, the acoustic impedance of each hole as a function of location was investigated. Neglecting the interaction between the holes, the overall acoustic impedance due to the perforations is expressed as a sum over all holes,

$$
z_{\text {perforation }}=\frac{1}{\sum_{n=1}^{N} \frac{\frac{r_{0}^{2} \pi}{R_{0}^{2} \pi}}{z_{\text {hole }}\left(R_{n}\right)},}
$$

where $n$ denotes the $n$th hole on the membrane surface, $R_{n}$ denotes the radial coordinate of the $n$th hole, and $N$ is the total number of holes. If $z_{\text {hole }}$ is uniform, as it is in Maa's model, Eq. (24) can be rewritten as

$$
z_{\text {perforation }}=\frac{1}{N \frac{\frac{r_{0}^{2} \pi}{R_{0}^{2} \pi}}{z_{\text {hole }}}}=\frac{z_{\text {hole }}}{\delta},
$$

which is consistent with Eq. (7).

The same example used in Sec. IIC is investigated in this section to demonstrate the effect of the membrane vibration on the overall impedance of the MPM. The overall acoustic impedance predicted by the presented model is compared to that predicted by Maa's model. The normalized resistances and reactances predicted by the rigid-walled model and the finite circular membrane of radius $R_{0}$ $=50 \mathrm{~mm}$ are shown in Figs. 4(a) and 4(b). The resistance predicted by Eq. (24) is lower than that predicted by Maa's rigid-wall model, while the reactance of this flexible wall model is higher than that of the rigid wall model above the fundamental resonance frequency. The most significant difference in the resistance and reactance is observed in the low frequency range near $200 \mathrm{~Hz}$. It could be concluded that the

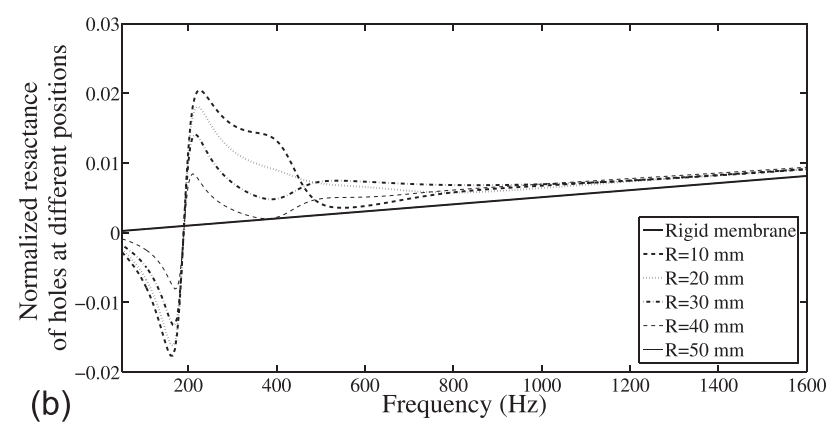

FIG. 3. Normalized resistance and reactance of a hole for five radial coordinates varying from $R=10$ to $R=50 \mathrm{~mm}$. 

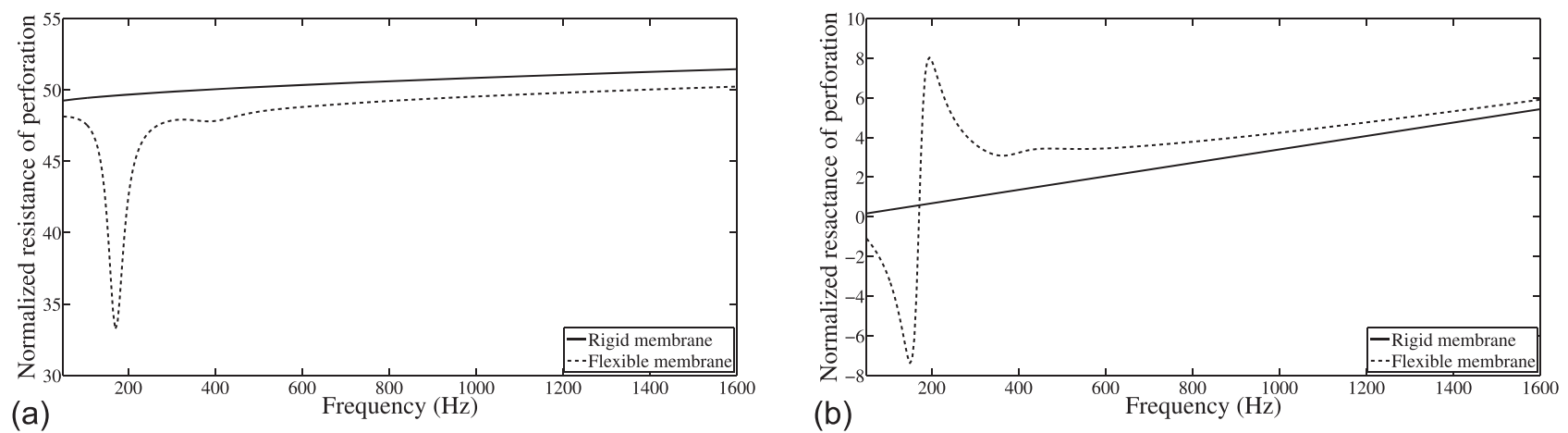

FIG. 4. Normalized resistance and reactance due to all of the holes on the MPM surface.

flexible wall assumption leads to a significant change in the acoustic impedance caused by the perforation, especially in the low frequency range.

Note that Eq. (24) considers only the acoustic impedance due to the perforations. To investigate the overall impedance of the MPM system, it is also necessary to consider the acoustic impedance of the membrane vibration. If the impedance of the perforation and that of the membrane vibration are known, then the overall impedance may be calculated using the electric-acoustic analogy, giving

$$
z_{\text {overall }}=\frac{1}{\frac{1}{z_{\text {perforation }}}+\frac{1}{z_{\text {vibration }}},}
$$

where $z_{\text {perforation }}$ denotes the impedance due to the perforations obtained by Eq. (24) and $z_{\text {vibration }}$ denotes the membrane vibration impedance given by Eq. (22). Once the overall impedance of the MPM system is obtained, the impedance of the MPM backed by an air cavity and a rigid wall is expressed as

$$
z=z_{\text {overall }}-j \cot \left(\frac{\omega D}{c_{0}}\right)
$$

where $D$ is the depth of the air cavity. Therefore, the sound absorption coefficient of an MPM with a backing cavity is given by

$$
\alpha=\frac{4 \operatorname{Re}(z)}{(1+\operatorname{Re}(z))^{2}+\operatorname{Im}(z)^{2}},
$$

where $\operatorname{Re}(z)$ and $\operatorname{Im}(z)$ are the real and imaginary parts of $z$, respectively.

\section{EXPERIMENTAL VALIDATION}

Sections III A-III C explore the experimental validation of the model derived in Sec. II. The experimental results are compared with the predicted results and the limitations of the assumptions used in the proposed model are also discussed.

\section{A. Experimental parameters}

To validate the model developed in this paper, sound absorption experiments were carried out in an impedance tube and at frequencies below the cutoff frequency to ensure plane wave incidence. The radius of the impedance tube was $R_{0}=50 \mathrm{~mm}$. The sound absorption coefficients of four commercially available MPMs were measured using the twomicrophone transfer function method (Chung and Blaser, 1980). The four MPMs were tested for two cavity depths $D$ of 25 and $50 \mathrm{~mm}$.

To predict the sound absorption of MPMs, it is crucial to measure the structural parameters of the MPMs, including the radius of the perforations $r_{0}$. The perforations of MPMs 1 to 3 were punched and the perforations were irregular polygons, unlike the circular perforations of MPM 4. The photomicrographs of the perforations of MPM 1 and MPM 4 are shown in Fig. 5 as examples. Because of the irregular geometry of the holes in MPMs 1 to 3, the equivalent hole radius $r_{0}$ needs to be estimated. The minimum, maximum, and average hole radius of MPMs 1 to 3 are shown in Table I for

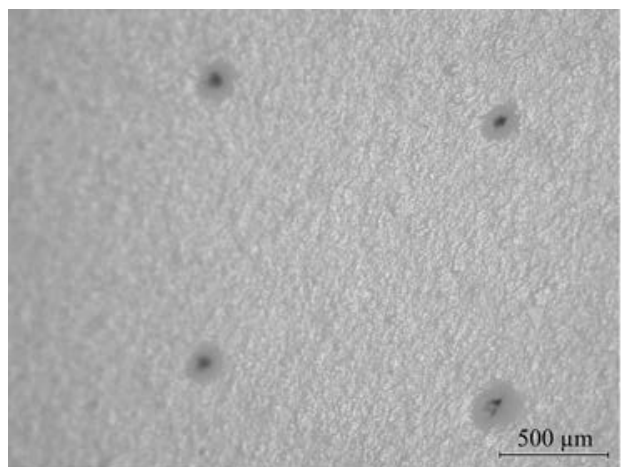

(a)

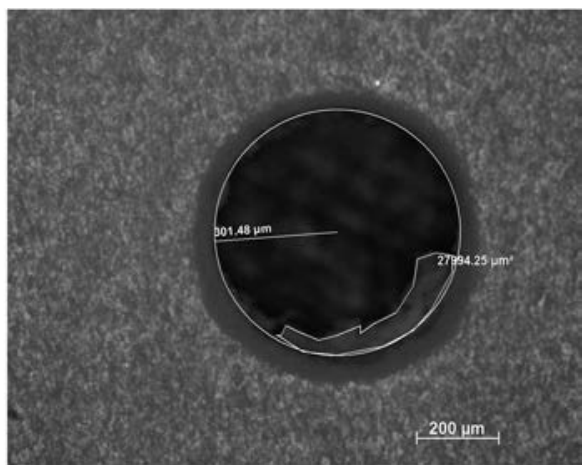

(b)
FIG. 5. Photomicrographs of perforations of MPM 1 and MPM 4. 
TABLE I. Measured hole radius of MPMs. The equivalent hole radius was determined from data fitting.

\begin{tabular}{lcccc}
\hline \hline MPM & $\begin{array}{c}\text { Minimum hole } \\
\text { radius } \\
r_{0 \min }(\mathrm{mm})\end{array}$ & $\begin{array}{c}\text { Maximum } \\
\text { hole radius } \\
r_{0 \max }(\mathrm{mm})\end{array}$ & $\begin{array}{c}\text { Average hole } \\
\text { radius } \overline{r_{0}}(\mathrm{~mm}) \\
\text { of ten holes }\end{array}$ & $\begin{array}{c}\text { Equivalent } \\
\text { hole radius } \\
r_{0}(\mathrm{~mm})\end{array}$ \\
\hline 1 & 0.016 & 0.030 & $0.026(\mathrm{std}=0.004)$ & 0.0226 \\
2 & 0.011 & 0.040 & $0.022(\mathrm{std}=0.008)$ & 0.0211 \\
3 & 0.009 & 0.042 & $0.029(\mathrm{std}=0.010)$ & 0.0248 \\
\hline \hline
\end{tabular}

10 randomly selected holes. The factor std is the standard deviation of the measured hole radius of each MPM.

Note that the perforations of MPM 4 were quite circular; however, in some cases the membrane material covered part of the hole area, as shown in Fig. 5(b). These areas need to be excluded during the calculation of the equivalent radius of MPM 4. Therefore, 20 holes on MPM 4 were randomly chosen and the equivalent radius measured from each photomicrograph was used to obtain an average equivalent radius for input to the analytical model.

The physical parameters of the MPMs were measured and are shown in Table II. The experimental results are compared to the predictions of the model presented in Sec. III B. It was not possible to directly measure the tension $T$ and the damping ratio $\eta$ by experiments. Hence, the equivalent value set $T, \eta$, and $r_{0}$ used in the analytical model have been obtained from the experimental measurements by fitting the measured data to the model using the optimization toolbox in MATLAB under a number of constraints (Waltz et al., 2006): positive tension; damping ratio less than 0.05; and hole radius varying from the minimum measured value $r_{0 \text { min }}$ to the maximum measured value $r_{0 \max }$ (listed in Table I). The constrained non-linear optimization was based on the subspace trust region method. The obtained values for $T, \eta$, and $r_{0}$ are shown in Figs. 6 and 7.

\section{B. Experimental results and discussions}

The experimental results of the four MPMs with an air cavity depth of $D=25 \mathrm{~mm}$ are shown in Fig. 6 and those for $D=50 \mathrm{~mm}$ are shown in Fig. 7. These experimental results are compared to the prediction results of three models: Maa's classic rigid wall model [Eq. (7)]; the model of a membrane absorber without perforation [Eq. (22)], and the presented model [Eq. (26)]. In Figs. 6 and 7, the dasheddotted curves are the prediction results of Maa's model, which neglects the membrane vibration effect; the prediction results for an unperforated membrane are shown as dotted

TABLE II. Measured MPM parameters.

\begin{tabular}{|c|c|c|c|c|c|}
\hline MPN & $\begin{array}{c}\text { Surface } \\
\text { density } \\
\rho_{\mathrm{p}}\left(\mathrm{kg} / \mathrm{m}^{2}\right)\end{array}$ & $\begin{array}{c}\text { Hole } \\
\text { radius } \\
r_{0}(\mathrm{~mm})\end{array}$ & $\begin{array}{c}\text { Membrane } \\
\text { thickness } \\
h(\mathrm{~mm})\end{array}$ & $\begin{array}{c}\text { Equivalent distance } \\
\text { between hole } \\
\text { centres } b(\mathrm{~mm})\end{array}$ & $\begin{array}{l}\text { Perforation } \\
\text { ratio } \delta(\%)\end{array}$ \\
\hline 1 & 0.2501 & 0.0226 & 0.17 & 1.32 & 0.092 \\
\hline 2 & 0.2503 & 0.0211 & 0.17 & 1.58 & 0.056 \\
\hline 3 & 0.2448 & 0.0248 & 0.17 & 1.63 & 0.073 \\
\hline 4 & 0.2506 & $\begin{array}{c}0.255 \\
(\operatorname{std}=0.031)\end{array}$ & 0.17 & 5.29 & 0.730 \\
\hline
\end{tabular}

curves; and the predictions obtained from the new model are shown as dashed curves. Furthermore, to verify the novelty of the proposed method, the proposed predictions of the four MPMs were compared with the method of Kang and Fuchs (1999) which is given by

$$
z_{\text {Kang and Fuchs }}=\frac{1}{\frac{1}{z_{\mathrm{Maa}}}+\frac{1}{1+j \omega \frac{\rho_{\mathrm{p}}}{\rho_{0} c_{0}}}} .
$$

The sound absorption coefficients of MPMs 1 to 3 predicted using Maa's model are low compared to the experimental results because the hole diameters are smaller than the range of applicability of Maa's model (roughly around $1 \mathrm{~mm}$ ), which consequently leads to high calculated normalized acoustic impedances. High normalized acoustic impedance is usually considered as the main reason for the effective sound absorption of micro perforated materials. However, Maa (2006) illustrated that when the normalized resistance of an MPP increases from one to five, its sound absorption coefficient decreases proportionally. Therefore, high resistances and low sound absorptions of MPMs are observed here using Maa's model due to the small perforations considered for MPMs 1 to 3 .

To assess the accuracy of the three models for prediction, the coefficient of determination $R_{\text {determination }}^{2}$ for each model is shown in Figs. 6 and 7, and is given by

$$
R_{\text {determination }}^{2}=1-\frac{\sum_{n_{\text {freq }}=1}^{N_{\text {freq }}}\left(\alpha_{\text {experiment }}-\alpha_{\text {prediction }}\right)^{2}}{\sum_{n_{\text {freq }}=1}^{N_{\text {freq }}}\left(\alpha_{\text {experiment }}-\overline{\alpha_{\text {experiment }}}\right)^{2}},
$$

where $n_{\text {freq }}$ denotes the index of the measured frequency, $N_{\text {freq }}$ denotes the total number of measured frequencies, $\alpha_{\text {prediction }}$ denotes the predicted sound absorption coefficient, and $\alpha_{\text {experiment }}$ denotes the measured sound absorption coefficient. As $R_{\text {determination }}^{2}$ approaches unity, the fidelity of the model increases.

For MPMs 1 to 4 , the $R_{\text {determination }}^{2}$ of the presented model is close to unity. Hence, the presented model provides a good agreement with the experimental results and is suitable for the prediction of the sound absorption of these MPMs. These results confirm that the new boundary condition theory and the derived equations are valid for these MPM samples. The negative $R_{\text {determination }}^{2}$ is because the error between the measured data and the predicted result is larger than the difference between the measured data and its mean. The negative $R_{\text {determination }}^{2}$ indicates the inaccuracy of the corresponding model.

It is noticed that there are small dips and peaks from 1200 to $1260 \mathrm{~Hz}$ in Figs. 6 and 7. They occur in the experimental results of every MPM. These are because of a structural resonance of the impedance tube itself.

When calculating the acoustic impedance due to the perforations of MPM 4, it was found that Eq. (7) underestimated 


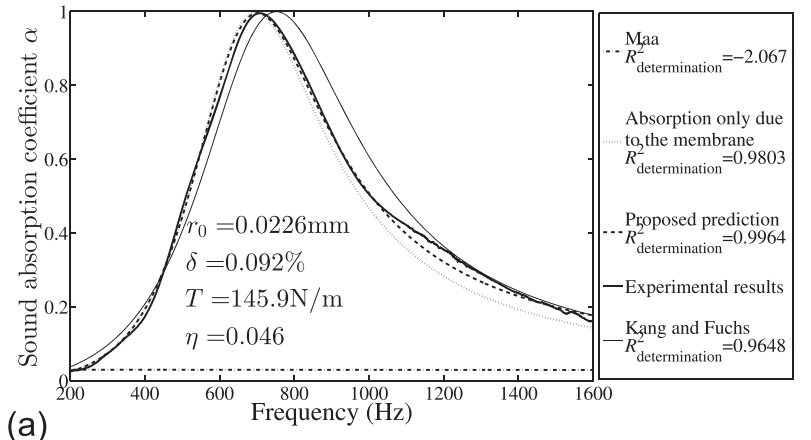

(a)

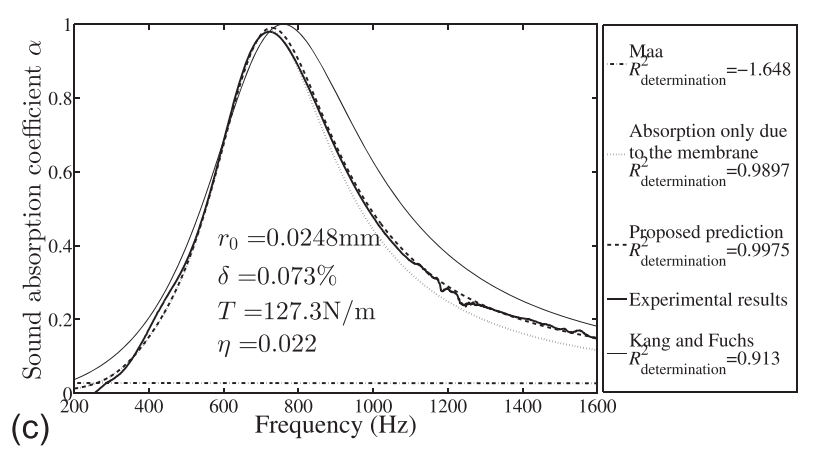

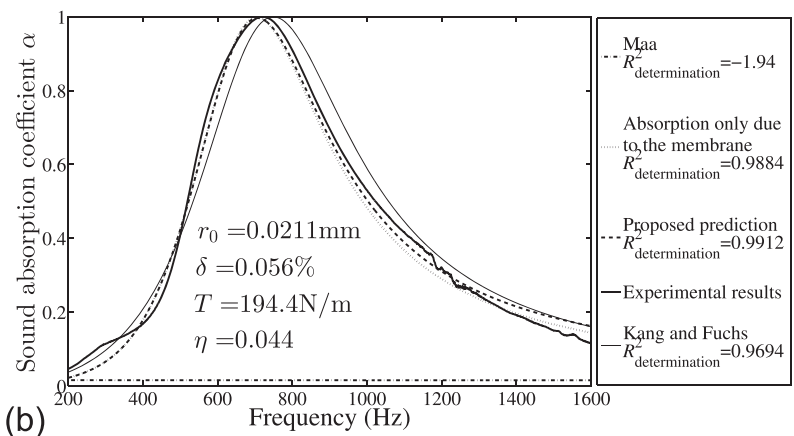

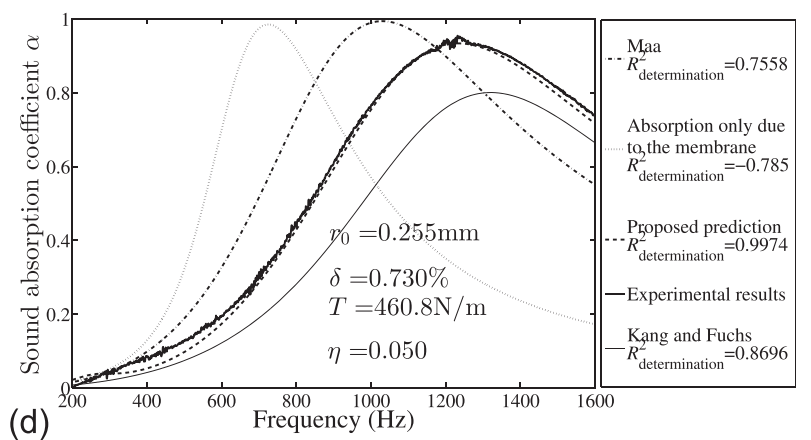

FIG. 6. Normal incidence sound absorption coefficients of MPMs 1 to 4 for $D=25 \mathrm{~mm}$.

the impedance due to the thermo-viscous friction. This has also been observed by Tayong et al. (2010), who used

$$
R_{s}=\frac{1}{2} \sqrt{2 \omega \rho_{0} \xi}
$$

to estimate the resistance due to the thermo-viscous friction, where $\xi$ is the dynamic viscosity. They added $4\left(R_{s} / \rho_{0} c_{0} \delta\right)$ to the normalized impedance due to the perforations. The value of
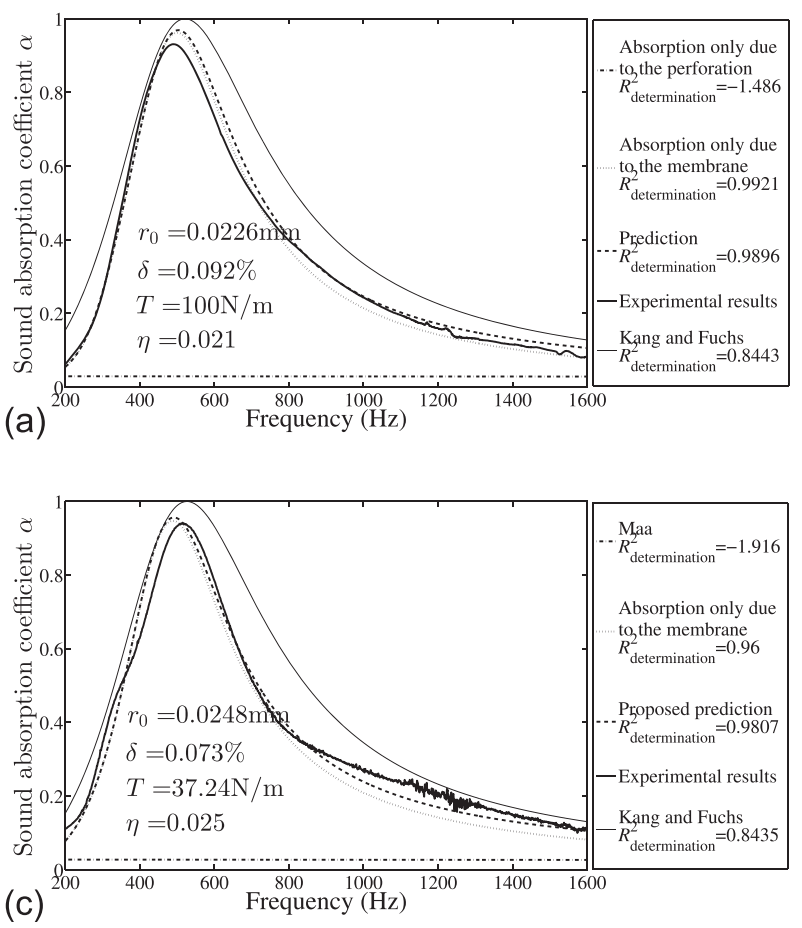

Eq. (31) is purely real and represents the resistance due to the thermo-viscous friction only. However, in Rayleigh's (1896) original derivation, $R_{s}$ was expressed as a complex value

$$
R_{s}=\frac{1}{2} \sqrt{2 \omega \rho_{0} \xi}(1+j)
$$

Therefore, in the presented model for MPM 4, Eq. (32) was used and $4\left(R_{s} / \rho_{0} c_{0} \delta\right)$ was added to Eq. (7). The
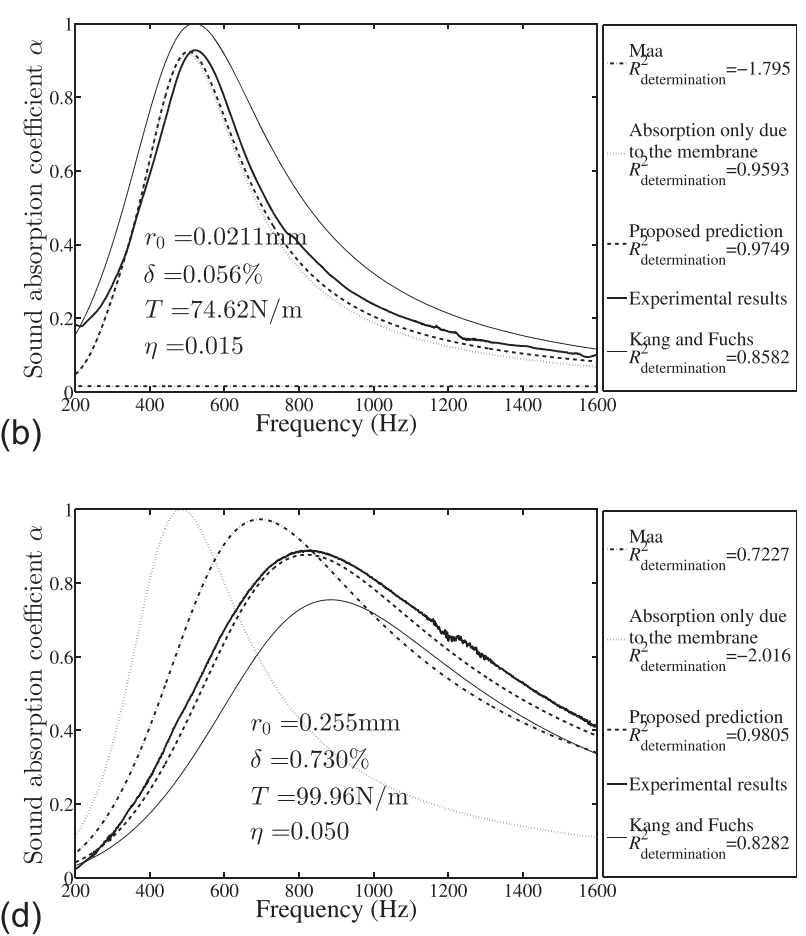

FIG. 7. Normal incidence sound absorption coefficients of MPMs 1 to 4 for $D=50 \mathrm{~mm}$. 


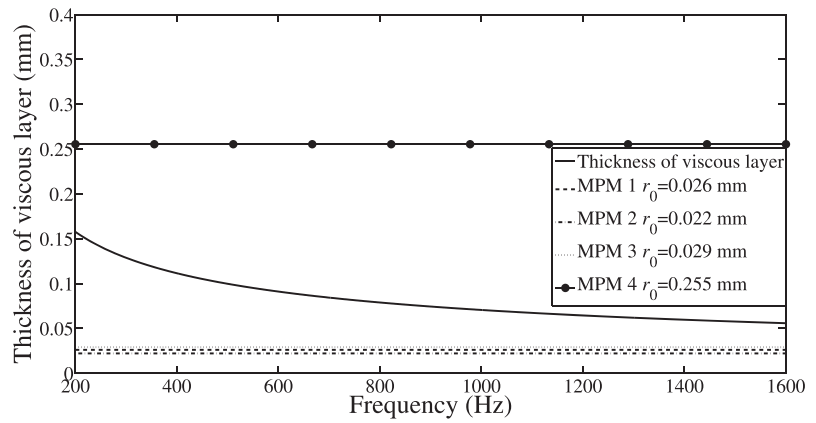

FIG. 8. Thickness of viscous layer $h_{\text {viscosity }}$ in the perforations compared with the hole radii of MPMs 1 to 4 .

prediction results agree with the measured results, as shown in Figs. 6(d) and 7(d).

Note that the additional impedance due to the thermoviscous friction is only of significance for the acoustic impedance of MPM 4. This can be ascribed to the significant difference between the hole radii of MPMs 1 to 3 and the hole radius of MPM 4. It is observed in Table II that the latter is 10 times larger than the former. The thickness of the viscous layer is defined by Maa (1975) as

$$
h_{\mathrm{viscosity}}=\sqrt{\frac{2 \times \mu}{\omega}},
$$

where for air $\mu=1.56 \times 10^{-5} \mathrm{~m}^{2} / \mathrm{s}$. As shown in Fig. 8, the hole radii of MPMs 1 to 3 are smaller than the thickness of the viscous layer such that the entire hole is covered by the viscous layer which limits the particle velocity in these holes. This results in high acoustic impedance of MPMs 1 to 3 and limited air flow through these holes. Hence, the thermo-viscous friction can be neglected. However, the hole radius of MPM 4 is larger than the thickness of the viscous layer and air can flow through the holes in MPM 4 more easily. The air flow through the holes forms a rotational jet and increases the impedance of the perforations. The flexibility of the membrane material can also contribute to the impedance of the thermo-viscous friction. Therefore, the additional impedance due to thermo-viscous friction was added to MPM 4 only and was neglected for the other three MPMs.

Comparing the experimental results of the MPMs, we could also conclude that the main absorption peaks of MPMs 1 to 3 are near the main absorption peaks of the membranes without perforation. As for MPM 4, the main absorption peak moves to the high frequency range and is near the main absorption peak for the predicted result of the rigid membrane model. This may imply that when the perforation is small as is the case for MPMs 1 to 3, the MPM absorption is mainly due to the membrane itself. In these cases, the perforations marginally broaden the sound absorption bandwidth but do not move the absorption peaks significantly. When MPM 4 is considered, the perforations are the main contributor to the sound absorption and the main absorption peak of the MPM is near the theoretical absorption peak due to the perforations.

In Figs. 6 and 7, the thin solid curves represent the predicted results based on the method of Kang and Fuchs
(1999). Their method is a simplified one which assumes that the acoustic impedance due to the membrane vibration is only dependent on the surface density of the membrane. The finite effect of the membrane vibration on the acoustic impedance is simplified by using a constant 1 in the term of $1+j \omega\left(\rho_{p} / \rho_{0} c_{0}\right)$, as seen in Eq. (29). Consequently, this model is less accurate than the proposed model which considers the response of a finite circular impervious membrane by solving its motion equation and optimizing the corresponding tension and damping. The mismatch of the experimental results and the predicted results using Kang and Fuchs's model validates this conclusion on the relative accuracy of the two models. Therefore, the proposed model is considered more accurate than the conventional Kang and Fuchs's model.

The structural parameters of MPMs 1 to 4 are all in a similar range of values, except that MPM 4 has a hole diameter an order of magnitude larger than the other three MPMs. The hole diameter and perforation ratio of MPM 4 are close to the structural parameters of a classic MPP, which is typically around $r_{0}=1 \mathrm{~mm}$ and $\delta=1 \%$. The impedance of the holes in MPM 4 is efficiently combined with the acoustic impedance due to the membrane vibration to offer effective sound absorption. On the contrary, the perforations in MPMs 1 to 3 are too small, and the acoustic impedance due to the perforations is thus too high to effectively contribute to the sound absorption. Extremely high acoustic impedance leads to a poor sound absorption from MPP/MPM absorbers (Maa, 2006).

Therefore, it is concluded that although the sound absorption bandwidths of MPMs 1 to 3 have been marginally broadened, the sound absorption properties of these MPMs are mainly governed by the membrane itself. Considering the expense of manufacturing the perforations, incorporating perforations of this size in commercial sound absorbing materials is likely to be ineffective. However, the sound absorption values obtained for MPM 4 indicate the effectiveness of such MPMs incorporating holes of suitable size. To achieve their optimum sound absorption, MPMs need to be carefully designed to effectively couple the membrane vibration impedance and the impedance due to the perforations. The presented theory is proposed as a tool to design such MPMs.

\section{Effect of perforation positions on the sound absorption of MPMs}

Based on the theory presented in Sec. II, it is logical to assume that since the membrane vibration affects the acoustic impedance of the perforations, the overall impedance and sound absorption properties of an MPM could be affected by the perforation positions since the vibration is not uniform over the membrane. By contrast, this presumption is different from the conventional theories which assume that the overall impedance of a flexible micro perforate (thin plate or membrane) is given by the coupled impedances of the material vibration and the perforations based on electric-acoustic analogy or average flow velocity. Hence, in the conventional theories, the overall impedance is independent of the position of the perforations. 
To validate this assumption, four additional MPMs were made using the same material as MPM 1. In Sec. III B, it was shown that MPM 1 can essentially be considered unperforated due to its low perforation ratio and small hole diameter.

The four additional MPMs may be categorized into two groups. In Group 1, each MPM was drilled with 73 holes, which hole radius $r_{0}=0.31 \mathrm{~mm}$. The holes were arranged at $R=45 \mathrm{~mm}$ for one of the manufactured MPMs and were evenly distributed over the membrane surface for the other. Therefore, in Group 1, the perforation ratios of two manufactured MPMs are identical and equal to $0.29 \%$. Similarly, for Group 2, each MPM was drilled with 48 holes. The hole radius $r_{0}$ is also $0.31 \mathrm{~mm}$. Therefore, the perforation ratio for each manufactured MPM in Group 2 is $0.19 \%$. The holes were at $R=45 \mathrm{~mm}$ for one of the MPMs and were evenly distributed for the other. In each group, the parameters of the MPMs are identical. Based on the conventional theories, their sound absorption coefficients should be identical; however, based on the proposed theory, their sound absorption coefficients might differ due to the hole positions, i.e., the effect of the membrane vibration on the acoustic impedance of the perforations.

Figure 9 shows the photograph of the four additional MPMs. The different perforation positions are illustrated in Fig. 9. The sound absorption measurements were conducted under the same experimental set up as described in Sec. III B. The measured sound absorption coefficients and the

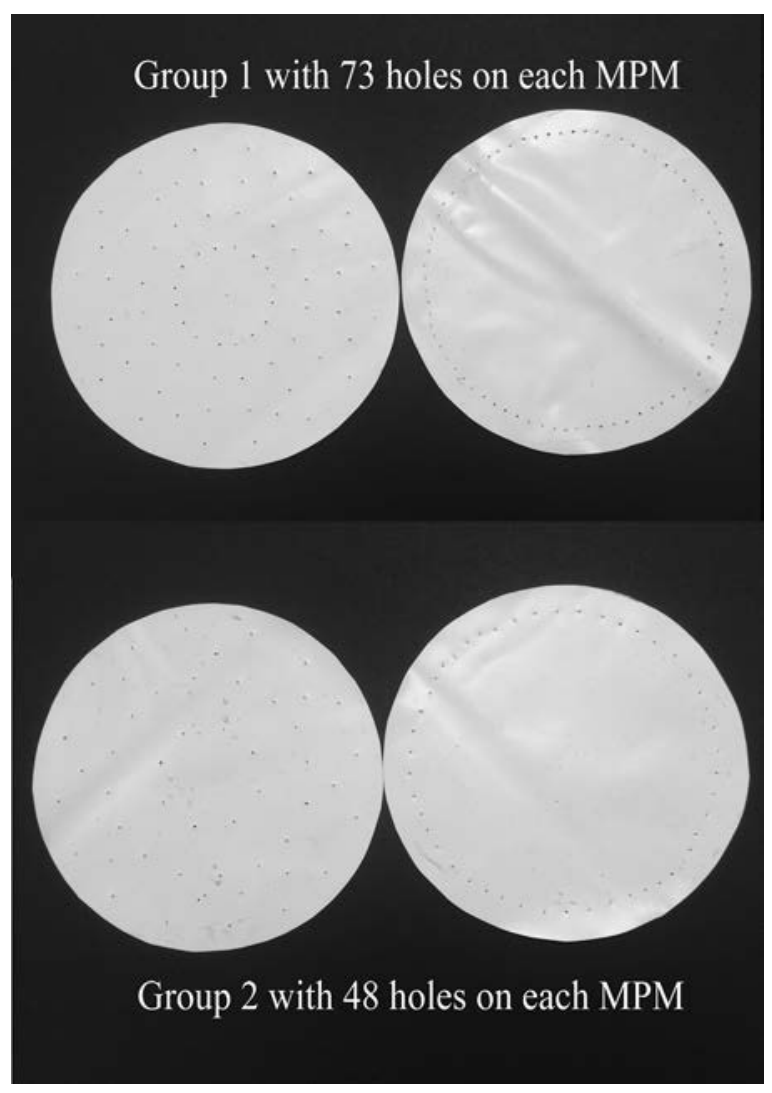

FIG. 9. Photograph of the additional MPMs made using the MPM 1 material. There are 73 and $48(0.31 \mathrm{~mm}$ holes $)$ drilled in each of the membranes, respectively.

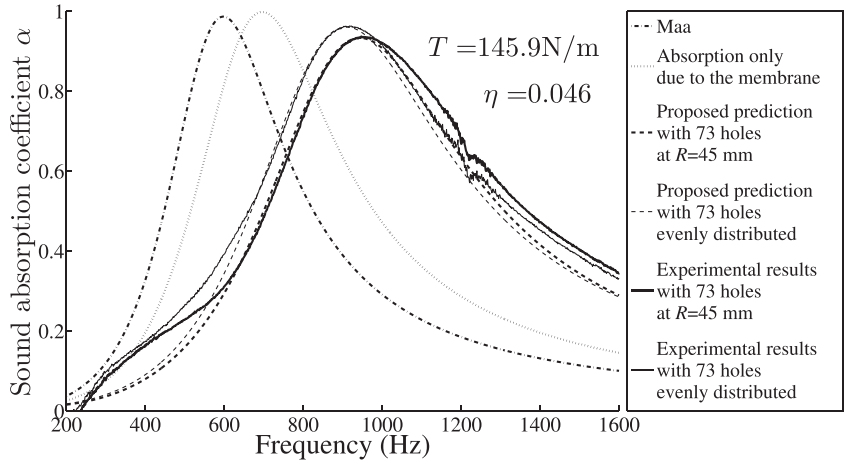

FIG. 10. Normal incidence sound absorption coefficients of the manufactured MPMs with 73 holes, $D=25 \mathrm{~mm}$.

predicted results based on the proposed method are compared for each group in Figs. 10 and 11.

Note that the values of the tension and damping are identical to those in Fig. 6(a) because it is assumed that the perforations have no effect on the mechanical properties of the membrane. The thick solid curves in Figs. 10 and 11 are the measured sound absorption coefficient curves for the manufactured MPMs with holes at $R=45 \mathrm{~mm}$ and the thin solid curves are those for the manufactured MPMs with holes evenly distributed. There are obvious differences between these curves which demonstrates the effect of the hole positions on the acoustic impedance of the MPMs, and hence supports the proposed theory. Moreover, the predicted curves are close to the experimental results for the corresponding manufactured MPMs, which suggested that the proposed theory is accurate.

\section{SUMMARY}

A new method for the prediction of the acoustic impedance and the sound absorption coefficient of a MPM is introduced in this paper. This method is based on a new boundary condition where the particle velocity at the hole wall boundary, which is assumed to be zero in Maa's theory, is assumed to be equal to the local membrane vibration velocity. By applying this new boundary condition to a circular membrane, it is shown that the acoustic impedance due to the perforation is affected by the membrane vibration and becomes a function of the membrane radial coordinates.

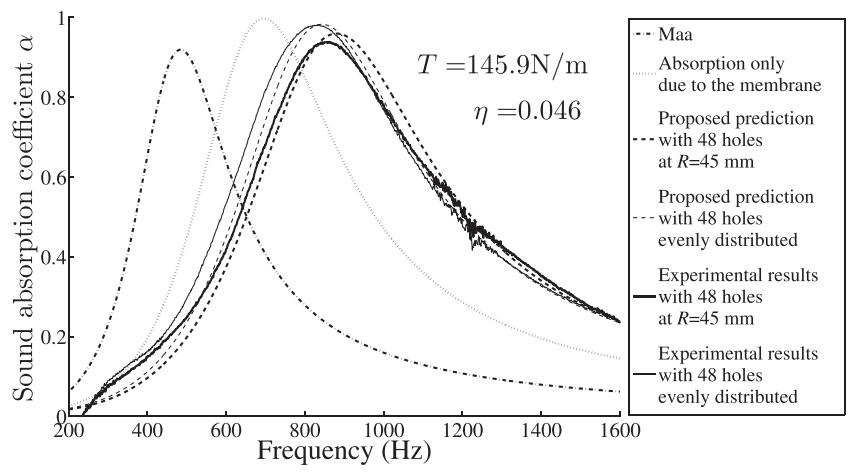

FIG. 11. Normal incidence sound absorption coefficients of the manufactured MPMs with 48 holes, $D=25 \mathrm{~mm}$. 
Using this new boundary condition, analytical models for the acoustic impedances of both the impedance associated with the perforations and the membrane vibration impedance are derived. The variability of the perforation impedance with hole location is investigated theoretically. The impedances due to the perforation and the membrane vibration are combined following the electric-acoustic analogy to present the overall impedance of the MPM. This new model is validated by experimental results for MPMs. Moreover, based on the proposed theory, it is validated experimentally that the hole position affects the acoustic impedance and sound absorption of MPMs, even if the MPM parameters, such as the hole radii, the thickness, and the perforation ratio, are identical.

This study extends the classic micro perforated theory and offers an accurate model for predicting the performance of flexible finite-sized MPMs. This study provides increased understanding of the coupling between the membrane vibration impedance and the impedance due to the perforations of MPMs.

\section{ACKNOWLEDGMENTS}

The authors wish to acknowledge the assistance of Dr. Erwin Gamboa for measuring the radii of the MPM samples. The authors are also very grateful to the staff of the electronic and mechanical workshops in the School of Mechanical Engineering at the University of Adelaide.

Bravo, T., Maury, C., and Pinhède, C. (2012). "Vibroacoustic properties of thin micro-perforated panel absorbers," J. Acoust. Soc. Am. 132, 789-798.

Burgemeister, K., and Hansen, C. (1996). "Calculating resonance frequencies of perforated panels," J. Sound Vib. 196, 387-399.

Chung, J., and Blaser, D. (1980). "Transfer function method of measuring in-duct acoustic properties. I. Theory," J. Acoust. Soc. Am. 68, 907-913.

Herdtle, T., Bolton, J. S., Kim, N. N., Alexander, J. H., and Gerdes, R. W. (2013). "Transfer impedance of microperforated materials with tapered holes," J. Acoust. Soc. Am. 134, 4752-4762.

Kang, J., and Fuchs, H. (1999). "Predicting the absorption of open weave textiles and micro-perforated membranes backed by an air space," J. Sound Vib. 220, 905-920.
Kinsler, L. E., Frey, A. R., Coppens, A. B., and Sanders, J. V. (1999). Fundamentals of Acoustics (John Wiley \& Sons, New York), pp. 82-92.

Lee, Y., Lee, E., and Ng, C. (2005). "Sound absorption of a finite flexible micro-perforated panel backed by an air cavity," J. Sound Vib. 287, 227-243.

Liu, J., and Herrin, D. (2010). "Enhancing micro-perforated panel attenuation by partitioning the adjoining cavity," Appl. Acoust. 71, 120-127.

Maa, D. Y. (1975). "Theory and design of microperforated panel sound absorbing constructions," Sci. Sin. 18, 55-71.

Maa, D. Y. (1997). "General theory and design of microperforated-panel absorbers," Acta Acust. 22, 385-393.

Maa, D. Y. (1998). "Potential of microperforated panel absorber," J. Acoust. Soc. Am. 104, 2861-2866.

Maa, D. Y. (2006). "Practical absorption limits of MPP absorbers," Acta Acust. 31, 481-484.

Park, S.-H. (2013). "Acoustic properties of micro-perforated panel absorbers backed by Helmholtz resonators for the improvement of low-frequency sound absorption,” J. Sound Vib. 332, 4895-4911.

Pfretzschner, J., Cobo, P., Simon, F., Cuesta, M., and Fernandez, A. (2006). "Microperforated insertion units: An alternative strategy to design microperforated panels," Appl. Acoust. 67, 62-73.

Rayleigh, J. W. S. B. (1896). The Theory of Sound (Macmillan and Co., New York), pp. 317-319.

Ruiz, H., Cobo, P., and Jacobsen, F. (2011). "Optimization of multiple-layer micro perforated panels by simulated annealing," Appl. Acoust. 72, 772-776.

Song, J., and Bolton, J. S. (2003). "Acoustical modeling of tensioned, permeable membranes," in INTER-NOISE and NOISE-CON Congress and Conference Proceedings (Institute of Noise Control Engineering, Washington, DC), pp. 965-970.

Takahashi, D., and Tanaka, M. (2002). "Flexural vibration of perforated plates and porous elastic materials under acoustic loading," J. Acoust. Soc. Am. 112, 1456-1464.

Tayong, R., Dupont, T., and Leclaire, P. (2010). "On the variations of acoustic absorption peak with particle velocity in micro-perforated panels at high level of excitation," J. Acoust. Soc. Am. 127, 2875-2882.

Toyoda, M., Mu, R. L., and Takahashi, D. (2010). "Relationship between Helmholtz-resonance absorption and panel-type absorption in finite flexible microperforated-panel absorbers," Appl. Acoust. 71, 315-320.

Walstijn, M. (2009). "Vibrations of strings and membranes," http://www. somasa.qub.ac.uk/mvanwalstijn/pbss/lectures/session5.pdf (Last viewed January 5, 2015).

Waltz, R. A., Morales, J. L., Nocedal, J., and Orban., D. (2006). “An interior algorithm for nonlinear optimization that combines line search and trust region steps," Math. Program. 107, 391-408.

Wang, C., Cheng, L., Pan, J., and Yu, G. (2010). "Sound absorption of a micro-perforated panel backed by an irregular-shaped cavity," J. Acoust. Soc. Am. 127, 238-246. 